\title{
Sleep Disorder in Children Suffering from Autism Spectrum Disorder in the Hilly Area of Uttarakhand
}

\author{
Ashok Kumar ${ }^{\circledR 1}$, Shweta Joshi ${ }^{\circledR 2}$, Sushil 0jha ${ }^{\circledR 3}$ \\ ${ }^{1}$ Associate Professor, Department of Pediatrics, GDMC, Dehradun, Uttarakhand, India, ${ }^{2}$ Tutor, Department of Pathology, GDMC, Dehradun, Uttarakhand, India, \\ ${ }^{3}$ Assistant Professor, Department of Ophthalmology, GDMC, Dehradun, Uttarakhand, India.
}

\section{Abstract}

Background: Autism Spectrum Disorder (ASD) includes neurodevelopmental problems throughout life that affect all areas of child development such as abnormal and impaired social interaction, problems in communication, restricted interest, repetitive and stereotypical behaviors. Sleep problems are most commonly complained by parents of children suffering from ASD. About 20-30\% of children less than 5 years with normal development give complaints of sleep problems mainly of bedtime resistance, sleep onset delay and night awakening. Less commonly reported sleep disturbance by parents is nightmares or night terrors repetitive and stereotypical behavior. Less frequently reported complain is of breathing sleep disorder. It has already been proved by many studies that ASD children have more complaints about sleep problems than in normal children. But the data of children with ASD having sleep problems in the Indian population is less, so with our study we want to know the prevalence of ASD children with sleep problems in children of the hilly area of Uttarakhand. Objective: The study aims to know the different problems related to sleep faced by children suffering from ASD ( Age range from 4 to 10 years ) with autistic spectrum disorders using the Children's Sleep Habit Questionnaire (Preschool and school-age, abbreviated version). Subjects and Method s: All patients of ASD presenting to the Psychiatry and Pediatric Outpatient department of Government Medical College and Susheela Tiwari trust Hospital, Haldwani, Uttarakhand from in February 2019 to January 2020. It's a cross-sectional study of children with different problems related to sleep faced by children suffering from ASD ( Age range from 4 to 10 years ) with autistic spectrum disorders using the Children's Sleep Habit Questionnaire (CSHQ) (Preschool and school-age, abbreviated version). Results: Demographic and clinical characteristics: The sample consisted of 30 children of ASD with sleep problems ( Mean Age 8.06 years, SD 3.25, Male 21 and Female 9 Children) age range from 4 Years to 10 Years and is compare from 30 normal children (mean age 9.06 Years, SD 3.15, Male 21 and Female 9) for the control group. Both groups were analysed using CSHQ tools. There were no statistically significant differences in age and gender ( $\mathrm{P}$ value more than 0.05$)$. About $60 \%(\mathrm{n}=18)$ of the children suffering from ASD had an intellectual disability and $40 \%(\mathrm{n}=12)$ children with ASD had normal Intellectual. Comparison of Total CSHQ score, bedtime resistance and sleep anxiety showed a statistically significant difference in children suffering from ASD with sleep disorder when compared from normal children. Using CSHQ tools sleep onset delay, night waking, day time sleepiness, parasomnias and sleep -disorder breathing does not show any statistical difference between children with ASD and normal children used as Control. Conclusion: This study compared sleep problems between children with ASD and typically developing children (4-10 Years of age) and examined associated factors of sleep problems in ASD from a different point of view.

Keywords: ASD, Autism Spectrum Disorder, CSHQ, Children's Sleep Habits Questionnaire

Corresponding Author: Shweta Joshi, Tutor, Department of Pathology, GDMC, Dehradun, Uttarakhand, India.

E-mail: drshweta1001@gmail.com

Received: 10 April 2020

Revised: 8 May 2020

Accepted: 17 May 2020

Published: 9 July 2020

\section{Introduction}

Autism Spectrum Disorder (ASD) includes neurodevelopmental problems throughout life that affect all areas of child development such as abnormal and impaired social interaction, problems in communication, restricted interest, repetitive and stereotypical behaviors. ${ }^{[1-4]}$ Armstrong KL et al, ${ }^{[5]}$ The sleep patterns of normal children the cause of insomnia in normal children are mainly behavioral but in cases of ASD it is mul- tifactorial.

Sleep problems are most commonly complained by parents of children suffering from ASD ranges from 40-80\%. About 20$30 \%$ children less than 5 years with normal development give complaints of sleep problems mainly of bedtime resistance, sleep onset delay and night awakening. Less commonly reported sleep disturbance by parents is nightmares or night terrors repetitive and stereotypical behavior. Less frequently 
reported complain is of breathing sleep disorder. ${ }^{[3]}$

The incidence of ASD is increasing in the last 20 Years. The increased incidence of ASD is more likely due to a change in the definition and diagnostic criteria of ASD. The present prevalence of ASD is around 1 child out of 88. ${ }^{[2]}$

Sleep problems are commonly reported by parents of young children. Approximately $25 \%$ of preschool-aged children with normal development have sleep problems about bedtime resistance, sleep onset delays, and night awakenings. Other less common sleep disturbances that concern parents include night terrors or nightmares and repetitive rhythmic behaviors, which manifest mostly during the toddler-preschool period in some children. Around $1-3 \%$ of children experience sleepdisordered breathing, a medical condition that also contributes to a reduced quality of sleep. ${ }^{[3]}$ Numerous research studies indicate that children with ASD experience more sleep problems than the general population. ${ }^{[4]}$ Current data on sleep problems in autism spectrum disorder is scarce, particularly from the Indian subcontinent. As there are cultural differences between west and Indian population it is yet to be ascertained if co-sleeping with parents as is common here in India play some role.

It has already been proved by many studies that ASD children have more complaints about sleep problems than in normal children. ${ }^{[4]}$ But the data of children with ASD having sleep problems in the Indian population is less, so with our study we want to know the prevalence of ASD children with sleep problems in children of the hilly area of Uttarakhand.

The study aims to know the different problems related to sleep faced by children suffering from ASD.

\section{Objective:}

The study aims to know the different problems related to sleep faced by children suffering from ASD (Age range from 4 to 10 years ) with autistic spectrum disorders using the Children's Sleep Habit Questionnaire (Preschool and schoolage, abbreviated version)

\section{Subjects and Methods}

All patients of ASD presenting to the Psychiatry and Pediatric Outpatient department of Government Medical College and Susheela Tiwari trust Hospital, Haldwani, Uttarakhand from in February 2019 to January 2020.

It's a cross-sectional study of children with different problems related to sleep faced by children suffering from ASD ( Age range from 4 to 10 years ) with autistic spectrum disorders using the Children's Sleep Habit Questionnaire (CSHQ) (Preschool and school-age, abbreviated version)

\section{Inclusion Criteria}

All children with ASD with complain of sleep problems (Age between 4-10 years)

\section{Exclusion Criteria}

- Age less than 4 years or more than 10 Year

- Any associated motor or sensory problems

- Any complaint of Seizure disorder or Organic brain problem or any chronic systemic disease

- If a patient is on known treatment which can alter sleep (e.g. Antiepileptic, Benzodiazepine or Barbiturates)

- Any Neuromuscular or Ear, Nose and Throat Problems which may alter sleep-associated breathing or Obstructive sleep apnea

- Parents not willing to answer questioner and consent forms for enrollment in the study

It's a cross-sectional study of children with different problems related to sleep faced by children suffering from ASD ( Age range from 4 to 10 years ) with autistic spectrum disorders using the Children's Sleep Habit Questionnaire (CSHQ) (Preschool and school-age, abbreviated version) with inclusion and exclusion criteria were enrolled in the study

Children's Sleep Habits Questionnaire (CSHQ): The CSHQ was used to assess sleep problems in children with ASD. It is a retrospective, 33-item abbreviated parent-report questionnaire that has been used in several studies to examine sleep behavior in young children. Parents are asked to recall sleep behaviors occurring over a "typical" recent week. Items are rated on a three-point scale: "usually" if the sleep behavior occurred five to seven times/week; "sometimes" for two to four times /week; and "rarely" for zero to one time/week. Some items are reversed to consistently make a higher score indicative of more disturbed sleep. CSHQ items are conceptually grouped into eight subscales reflecting the following sleep domains: 1) Bedtime Resistance, 2) Sleep Onset Delay, 3) Sleep Duration, 4) Sleep Anxiety, 5) Night Wakings, 6) Parasomnias, 7) Sleep- Disordered Breathing, 8) Daytime Sleepiness. Total Sleep Disturbance score includes all items of the eight subscales.

It should be noted that CSHQ is primarily a screening tool. The sleep domains reflected in seven of the CSHQ subscales do parallel symptom constellations associated with ICSD (revised) classifications that represent the most common sleep disorders in this age group, however, the CHSQ is not a diagnostic tool or used to define the underlying etiology of the presenting sleep symptoms. The utility of the various subscale scores on the CSHQ is in both alerting the clinician of a potential sleep disorder and providing information that would serve as a starting point for a more detailed clinical evaluation. The CSHQ has been translated into Hindi and validated for use with Indian children and has been shown to have good reliability and validity (Narendhran et al. 2008). 


\section{Statistical Analysis}

Descriptive statistics like Means and Standard deviations were calculated for age and other parameters.

The Total score on CSHQand 8 subscale scores was compared using $t$ and $F$ test

\section{Results}

\section{Demographic and clinical characteristics}

The sample consisted of 30 children of ASD with sleep problems ( Mean Age 8.06 years, SD 3.25, Male 21 and Female 9 Children) age range from 4 Years to 10 Years and is compare from 30 normal children (mean age 9.06 Years, SD 3.15, Male 21 and Female 9) for the control group. Both groups were analysed using CSHQ tools

There were no statistically significant differences in age and gender ( $\mathrm{P}$ value more than 0.05$)$. About $60 \%(\mathrm{n}=18)$ of the children suffering from ASD had an intellectual disability and $40 \%(\mathrm{n}=12)$ children with ASD had normal Intellectual. [Table 1]

Comparison of Total CSHQ score, bedtime resistance and sleep anxiety showed a statistically significant difference in children suffering from ASD with sleep disorder when compared from normal children [Table 2]

By Using CSHQ tools sleep onset delay, night waking, day time sleepiness, parasomnias and sleep -disorder breathing does not show any statistical difference between children with ASD and normal children used as Control [Table 3]

\section{Discussion}

Sleep problems are most commonly complained by parents of children suffering from ASD ranges from $40-80 \%$. About $20-$ $30 \%$ of children less than 5 years with normal development give complaints of sleep problems mainly of bedtime resistance, sleep onset delay and night awakening.

Armstrong KL et al, ${ }^{[5]}$ The sleep patterns of normal children the cause of insomnia in normal children is mainly behavioral but in cases of ASD it is multifactorial

Richdale and Prior et al, ${ }^{[3]}$ compared preschool- and schoolaged children with ASD to a normative sample and found significantly higher levels of current general sleep difficulties according to parent report ( $44 \%$ versus $27 \%$ ). Parents of autistic children commonly express that their children resist going to bed at an appropriate time, have difficulties in initiating sleep, and exhibit night time, and early morning waking. ${ }^{[3]}$ There is a component of poor sleep hygiene leading to sleep problems.

A study done by Flavia Cortesi et al, ${ }^{[4]}$ showed that sleep problems are the most common associated complaints of children suffering from ASD. Although children with ASD experience similar sleep problems as in a child with normal development but some studies suggest the prevalence is markedly higher than expected. Similar to our study, They showed demonstrated Sleep onset and maintenance problems resulting in reduced sleep duration are the most common concerns expressed by the parents of children with ASD. ${ }^{[4]}$ Regulation of sleep in children with ASD is poorly understood. They suggested that there is a bifurcation of the sleep/wake cycle with increased sensitivity to external noise and short sleep duration causing irregular sleep onset and wake up times. Also, children with ASD have an altered balance between homeostatic and circadian forces leading to heightened sensitivity of the sleep/wake cycle to environmental stimuli.

Sleep regulation tended to improve with age. ${ }^{[6]}$ A study shows that more than $70 \%$ of children with ASD experience delays in circadian rhythm development by at least 5 months compared to typically developing children. ${ }^{[7]}$

Goodlin-Jones et al found that children with autism sleep for shorter periods during a 24-hour day. This suggests that sleep patterns in children with autism are organized differently than other children. ${ }^{[8]}$

Regarding the relationship between IQ and sleep problems, Richdale and Prior et al, ${ }^{[9]}$ reported more frequent sleep problems in children with moderate to severe intellectual disability compared to autistic children with mild degrees of disability. It is noted that children with mental retardation have found more night waking than children with autism and no retardation.

A positive association has been noted between autism severity and parent-reported sleep problems. These findings suggest that sleep problems are part of the autism symptom complex and increase with increasing autism severity. ${ }^{[10-15]}$

Many studies have suggested that difficulties with sleep onset and maintenance and with reduced sleep duration are the most common sleep disorders in children with ASD. ${ }^{[16-19]}$ Other sleep disturbances in ASD include parasomnias like sleepwalking, night terrors, and sleep-related movement disorders as well as bedtime resistance. ${ }^{[8,16]}$ The limitation of our study is that we have taken the observation of parents.

Sleep patterns in children with intellectual disabilities have been investigated in several studies. ${ }^{[13-15]}$ In these studies, children with intellectual disabilities were more likely to have high sleep problems than healthy individuals, and more severe levels of intellectual disability were associated with more sleep difficulties. ${ }^{[13-15]}$ However, in the ASD group alone, there are only a few reports about the relationship between cognitive development and sleep problems. ${ }^{[13,17]}$ We found no significant differences between ASD patients with normal intellectual capacity and intellectual disability regarding sleep disturbances. This result was consistent with previous reports 


\begin{tabular}{|c|c|c|c|}
\hline & & Case, $\mathbf{N}=\mathbf{3 0}$ & Controls, $\mathbf{N}=\mathbf{3 0}$ \\
\hline Mean Age (in year) & & 8.06 & 9.01 \\
\hline Intellectual capacity ( $\mathrm{N} \%)$ & $\begin{array}{l}\text { Normal }(n=12 \quad) \text { Intellectual } \\
\text { disability }(n=18)\end{array}$ & 4060 & 100 \\
\hline
\end{tabular}

Table 2: The comparison of CSHQ scores between the patient and control groups (Statistically significant score $P$ value less than 0.05 )

\begin{tabular}{|c|c|c|c|}
\hline CSHQ & $\begin{array}{l}\text { ASD Patients, } \quad \mathrm{N}=\mathbf{3 0} \\
\text { Mean (SD) }\end{array}$ & Controls, $\mathbf{N}=30$ Mean(SD) & P-Value \\
\hline Total score & 53 & 50 & Less than 0.05 \\
\hline Sleep anxiety & 6.30 & 5.60 & Less than 0.05 \\
\hline
\end{tabular}

\begin{tabular}{lllc}
\hline Table 3: The comparison of CSHQ scores between the patient and control groups (Statistically significant score P-value Mor than 0.05) \\
\hline CSHQ & $\begin{array}{l}\text { ASD Patients, N=30 Mean } \\
\text { (SD) }\end{array}$ & Controls, $\mathbf{N = 3 0}$ Mean(SD) & P-Value \\
& 6.0 & 6.2 & More than 0.05 \\
Sleep onset delay & 4.08 & 3.90 & More than 0.05 \\
Night waking & 13.40 & 13.38 & More than 0.05 \\
Daytime sleepiness & 9.0 & 8.72 & More than 0.05 \\
parasomnias & 3.55 & 3.46 & More than 0.05 \\
\hline Sleep-disorder breathing & & & \\
\hline
\end{tabular}

indicating that sleep disorders can appear at all IQ levels. ${ }^{[13,17]}$ In light of these findings, it can be speculated that sleep problems in ASD can occur independently of intellectual disability.

\section{Conclusion}

This study compared sleep problems between children with ASD and typically developing children (4-10 Years of age) and examined associated factors of sleep problems in ASD from a different point of view. Comparison of Total CSHQ score, bedtime resistance and sleep anxiety showed a statistically significant difference in children suffering from ASD with sleep disorder when compared from normal children.

Sleep disorders may negatively affect the autistic symptoms and inattentiveness in ASD children.

The successful intervention for these sleep problems may prevent functional impairments and improve the clinical symptoms and quality of life of both patients and parents.

\section{Limitations}

The current study has several limitations. The cross-sectional nature of the study indicates a limitation. Therefore, it is necessary to interpret the results carefully. The second limitation is that the age range is relatively small (4-10 Years). The types and severity of sleep disorders may vary in different age groups. Another limitation is sample size which is 30 and cannot be extrapolated to all patients of ASD. So the author recommends a larger sample size to conclusively comment on the type of sleep disturbance and also any effect of sex on it.

\section{References}

1. Faras H, Ateeqi NA, Tidmarsh L. Autism spectrum disorders. Ann Saudi Med. 2010;30(4):295-300. Available from: https: //dx.doi.org/10.4103/0256-4947.65261.

2. Prevalence of autism spectrum disorders-Autism and Developmental Disabilities Monitoring Network, 14 sites, United States, 2008. MMWR Surveill Summ. 2012;61(3):1-19.

3. Krakowiak P, Goodlin-Jones B, Hertz-Picciotto I, Croen LA, Hansen RL. Sleep problems in children with autism spectrum disorders, developmental delays, and typical development: a population-based study. J Sleep Res. 2008;17(2):197-206. Available from: https://dx.doi.org/10.1111/j.1365-2869.2008. 00650.x.

4. Cortesi F, Giannotti F, Ivanenko A, Johnson K. Sleep in children with autistic spectrum disorder. Sleep Med. 2010;11(7):659-664. Available from: https://dx.doi.org/10. 1016/j.sleep.2010.01.010. 
5. Armstrong KL, Quinn RA, Dadds MR. The sleep patterns of normal children. Med J Aust. 1994;161(3):202-205. Available from: https://dx.doi.org/10.5694/j.1326-5377.1994. tb127383.x.

6. Matsuura H, Tateno K, Aou S. Dynamical properties of the two-process model for sleep-wake cycles in infantile autism. Cogn Neurodynamics. 2008;2(3):221-228. Available from: https://dx.doi.org/10.1007/s11571-008-9051-3.

7. Segawa M. Epochs of development of the sleep-wake cycle reflect the modulation of the higher cortical function particular for each epoch. Sleep Biol Rhythms. 2006;4(1):4-15. Available from: https://dx.doi.org/10.1111/j.1479-8425.2006.00205.x.

8. Goodlin-Jones BL, Tang K, Liu J, Anders TF. Sleep Patterns in Preschool-Age Children With Autism, Developmental Delay, and Typical Development. J Am Acad Child Psy. 2008;47(8):930-938. Available from: https://dx.doi.org/10. 1097/chi.0b013e3181799f7c.

9. Richdale AL, Prior MR. The sleep/wake rhythm in children with autism. Eur Child Adoles Psy. 1995;4(3):175-186. Available from: https://dx.doi.org/10.1007/bf01980456.

10. Schreck K. Sleep problems as possible predictors of intensified symptoms of autism*1. Res Dev Disabil. 2004;25(1):57-66. Available from: https://dx.doi.org/10.1016/j.ridd.2003.04.007.

11. Mayes SD, Calhoun SL. Variables related to sleep problems in children with autism. Res Autism Spectr Disord. 2009;3(4):931-941. Available from: https://dx.doi.org/10. 1016/j.rasd.2009.04.002.

12. DeVincent CJ, Gadow KD, Delosh D, Geller L. Sleep Disturbance and its Relation to DSM-IV Psychiatric Symptoms in Preschool-Age Children with Pervasive Developmental Disorder and Community Controls. J Child Neurol. 2007;22(2):161-169. Available from: https://dx.doi.org/10. 1177/0883073807300310.

13. Krakowiak P, Goodlin-Jones B, Hertz-Picciottio I, Croen LA, Hansen RL. Sleep problems in children with autism spectrum disorders, developmental delays, and typical development: a population-based study. J Sleep Res. 2008;17(2):197-206. Available from: https://dx.doi.org/10.1111/j.1365-2869.2008. 00650.x.
14. Didden R, Korzilius H, van Aperlo B, van Overloop C, de Vries M. Sleep problems and daytime problem behaviours in children with intellectual disability. J Intellect Disabil Res. 2002;46(7):537-547. Available from: https://dx.doi.org/10. 1046/j.1365-2788.2002.00404.x.

15. Richdale A, Francis A, Gavidia-Payne S, Cotton S. Stress, behaviour, and sleep problems in children with an intellectual disability. Am J Intellect Dev Disabil. 2009;25(2):147-161.

16. Cohen S, Conduit R, Lockley SW, Rajaratnam SM, Cornish KM. The relationship between sleep and behavior in autism spectrum disorder (ASD): a review. J Neurodev Disord. 2014;6(1). Available from: https://dx.doi.org/10.1186/18661955-6-44.

17. Hirata I, Mohri I, Kato-Nishimura K, Tachibana M, Kuwada A, Kagitani-Shimono K, et al. Sleep problems are more frequent and associated with problematic behaviors in preschoolers with autism spectrum disorder. Res Dev Disabil. 2016;49-50:86-99. Available from: https://dx.doi.org/10.1016/j.ridd.2015.11.002.

18. Herrmann S. Counting Sheep: Sleep Disorders in Children With Autism Spectrum Disorders. J Pediatr Health Care. 2016;30:143-154.

19. Wang G, Liu Z, Xu G. Sleep Disturbances and Associated Factors in Chinese Children with Autism Spectrum Disorder: A Retrospective and Cross-Sectional Study. Child Psychiatry Hum Dev. 2016;47:248-258.

Copyright: (C) the author(s), 2020. It is an open-access article distributed under the terms of the Creative Commons Attribution License (CC BY 4.0), which permits authors to retain ownership of the copyright for their content, and allow anyone to download, reuse, reprint, modify, distribute and/or copy the content as long as the original authors and source are cited.

How to cite this article: Kumar A, Joshi S, Ojha S. Sleep Disorder in Children Suffering from Autism Spectrum Disorder in the Hilly Area of Uttarakhand. Asian J. Clin. Pediatr. Neonatol. 2020;8(2):1-5.

DOI: dx.doi.org/10.47009/ajcpn.2020.8.2.1

Source of Support: Nil, Conflict of Interest: None declared. 